\title{
The impact of the Covid-19 pandemic on the short and mid-term urological emergencies and the emergency department
}

\author{
Hülya Yılmaz BAŞER ${ }^{1}$, Aykut BAŞER ${ }^{2, *}$ (i)
}

${ }^{1}$ Department of Emergency Medicine, Hitit University Erol Olçok Education and Research Hospital, Çorum, Turkey

${ }^{2}$ Department of Urology, Hitit University School of Medicine, Çorum, Turkey

\begin{abstract}
Received: 05.03 .2021
Accepted/Published Online: 25.03 .2021

$\bullet$

Final Version: 30.08.2021

Abstract

Dynamic changes are observed in the delivery of health care services due to the COVID-19 Pandemic. Its effect in the short term is a dramatic decrease in service, however, its effect in the medium and long term is unknown. In this study, we aimed to investigate the effects of the COVID-19 pandemic on emergency department and emergency urological surgery in the short and medium term during the 8-month period, and the reasons for possible changes. Emergency department operations, urology operations and emergency surgical procedures between April and November were compared as the 2020 pandemic period and the 2019 non-pandemic period. The relevant information was obtained from the hospital management information system. Descriptive analysis and statistical methods comparing the two periods were used. In the early stages of the pandemic, significant decreases were observed in both urology procedures and emergency department operations. In the medium period, while emergency department operations and urology consultations returned to the non-pandemic periods, there was no such a change in in emergency urological surgeries. In the medium-term effects of the pandemic, emergency department operations returned to the non-pandemic periods due to reasons such as patients' abuse of emergency departments in line with their requests for rapid diagnosis and treatment. In accordance with the changing nature of the pandemic, it is necessary to make different scheduling for emergency department operations and emergency surgeries.
\end{abstract}

Keywords: COVID-19, emergency department, pandemic, urological emergencies

\section{Introduction}

At the end of 2019, the coronavirus disease (COVID-19) caused by the Severe Acute Respiratory Syndrome Coronavirus 2 (SARS-CoV-2), which started in Wuhan, China and affected the whole world, was defined (1-3). With the virus affecting the whole world, the World Health Organization (WHO) named this disease COVID-19 and declared it as a pandemic (4). In this process, the first case in our country was seen on March 11, 2020 (4). Following the declaration of pandemic, increases were observed in the density of hospitals. For the patient density in hospitals observed all over the world, healthcare systems implemented significant changes to maintain the delivery of primary health care services and reduce the risk of collapse $(5,6)$. The Republic of Turkey Ministry of Health, which is the national health authority in Turkey, determined an action plan by declaring all national and private hospitals that fulfilled predefined requirements as 'Pandemic Hospitals' at the end of March. While Pandemic Hospitals mainly served COVID-19 patients, they also continued routine emergency service delivery and emergency surgeries.

Our aim in this study was to examine the short and medium term effects of the pandemic by comparing the emergency department operations and the change in urology health service delivery with the same period of 2019, especially the number of patients admitted to the emergency department of our hospital, which is the only tertiary care hospital in the province where it is located and continued to provide both pandemic patients and other health services during the pandemic, during the pandemic period.

\section{Materials and methods}

\subsection{Study type}

This study was a retrospective observational study and was approved by the local ethics committee (approval number; 369).

\subsection{Data source and population}

Our hospital is the only tertiary care hospital serving approximately 530.000 people in the province where we live. Its emergency department is a clinic where healthcare services are provided uninterruptedly, and adult and pediatric emergency services are separate. All procedures in our hospital (such as patient application, examination note, requested examinations, interventions, and consultations) are recorded in the hospital information management system. In our retrospective observational study in which the data were obtained from the hospital information management system, the data showing the operations of the emergency and urology 
departments in an 8-month period between April 1 and November 31, 2020, were retrospectively analyzed. Then, the data were compared with the non-pandemic period of 2019 , which included the same 8-month period. The emergency department of our hospital provides emergency services for adults and children. Since pediatric emergency urological patients are examined by pediatric urology, emergency admissions under the age of 18 were excluded from the study.

\subsection{Definitions}

- Application to Emergency Department Outpatient; refers to the number of patients aged 18 and over, admitted to the emergency department,

- Application to Urology Outpatient; refers to the number of patients admitted to the urology outpatient clinics,

- Number of urology consultations; refers to the number of emergency consultations requested from the emergency department to the urology department,

- Percentage of urology consultation; refers to the ratio of the number of emergency consultations requested from the emergency department to the urology department to the number of patients admitted to the emergency department,

- Total number of urological surgeries; refers to total number of surgeries performed by urology,

- The Number of emergency urological surgeries; refers to the number of emergency surgeries performed by urology,

- Percentage of emergency use of the Urology Operating Room; refers to the ratio of the number of emergency urological surgeries to the total number of urological surgeries,

- Percentage of emergency urological surgery; refers to the percentage of the ratio of the number of emergency surgeries performed by urology to the number of patients admitted to the emergency department.

\subsection{Statistical analysis}

The data were analyzed using the SPSS 22 package program. The normal distribution of data was tested by the Kolmogorov-Smirnov/Shapiro-Wilks test. Number and percentage expressions were used for descriptive statistics. Mann Whitney-U tests were used to compare 2019 and 2020. $\mathrm{p}<0.05$ was considered statistically significant.

\section{Results}

There was a decrease of $31.05 \%$ in the admissions to the emergency department of our hospital, which is the only tertiary care hospital in the province where it is located, in the 8-month period from the month following the declaration of the pandemic compared to the previous year. A similar situation was also observed with a decrease of $67.07 \%$ in the admissions to the urology outpatient clinic during the same period. There was a statistically significant change in the number of applications to emergency department outpatient and application to urology outpatient $(\mathrm{p}=0.006, \mathrm{p}=0.001$, respectively). An increase of $8.37 \%$, which was not statistically significant, was observed in the number of urology consultations $(p=0.674)$. A significant increase of $57.14 \%$ was found in the percentage of urology consultation $(p=0.036)$. When the surgical procedures performed during the pandemic period were examined, the total number of urological surgeries decreased by $42.14 \%$, however, the number of emergency urological surgeries increased by $33.3 \% \quad(p=0.001, \quad p=0.130, \quad$ respectively $)$ While the percentage of emergency urological surgery was $0.087 \%$ in 2019 , it increased to $0.185 \%$ with an increase of $112.64 \%$ in $2020(p=0.016)$, and it was observed that the percentage of emergency use of the urology operating room increased from $2.19 \%$ to $5.06 \%$ with an increase of $131.05 \%(\mathrm{p}=0.016)$ (Table 1).

Table 1. Change of emergency and urology department parameters during pandemic and non-pandemic periods

\begin{tabular}{|l|c|c|c|c|}
\hline & $\begin{array}{c}\text { Non- } \\
\text { pandemic } \\
\text { Period } \\
\text { (2019 Year) }\end{array}$ & $\begin{array}{c}\text { Pandemic } \\
\text { Period } \\
\text { (2020 } \\
\text { Year) }\end{array}$ & $\begin{array}{c}\text { Variation } \\
(\%)\end{array}$ & $p$ \\
\hline $\begin{array}{l}\text { Application to } \\
\text { emergency } \\
\text { department } \\
\text { outpatient (n) }\end{array}$ & 345393 & 238156 & -31.05 & 0.006 \\
\hline $\begin{array}{l}\text { Application to } \\
\text { urology outpatient } \\
\text { (n) }\end{array}$ & 26515 & 10321 & -67.07 & 0.001 \\
\hline $\begin{array}{l}\text { Number of urology } \\
\text { consultations (n) }\end{array}$ & 490 & 531 & +8.37 & 0.674 \\
\hline $\begin{array}{l}\text { Total number of } \\
\text { urological surgeries } \\
\text { (n) }\end{array}$ & 1775 & 1027 & -42.14 & 0.001 \\
\hline $\begin{array}{l}\text { The number of } \\
\text { emergency } \\
\text { urological surgeries } \\
\text { (n) }\end{array}$ & 39 & 52 & +33.3 & 0.130 \\
\hline $\begin{array}{l}\text { Percentage of } \\
\text { urology consultation } \\
\text { (\%) }\end{array}$ & 0.14 & 0.22 & +57.14 & 0.036 \\
\hline $\begin{array}{l}\text { Percentage of } \\
\text { emergency } \\
\text { urological surgery } \\
\text { (\%) }\end{array}$ & 0.087 & 0.185 & +112.64 & 0.016 \\
\hline $\begin{array}{l}\text { Percentage of } \\
\text { emergency use of } \\
\text { the urology } \\
\text { operating room (\%) }\end{array}$ & 2.19 & 5.06 & +131.05 & 0.016 \\
\hline & & & & \\
\hline & & & & \\
\hline
\end{tabular}




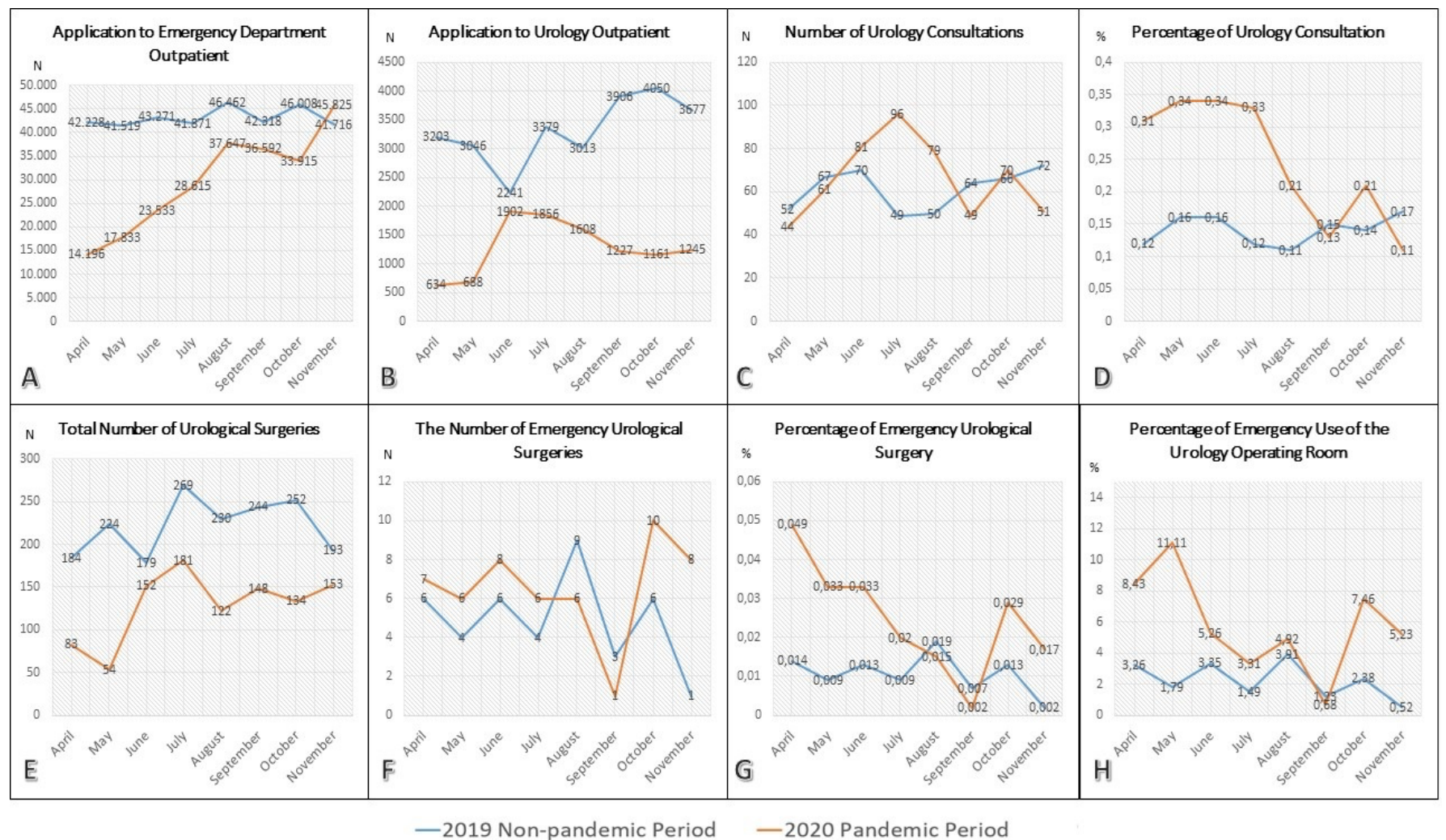

Fig. 1. A. Month-by-month change of application to emergency department outpatient during pandemic and non-pandemic periods. B. Monthby-month change of application to urology outpatient during pandemic and non-pandemic periods. C. Month-by-month change of number of urology consultations during pandemic and non-pandemic periods. D. Month-by-month change of percentage of urology consultations during pandemic and non-pandemic periods. E. Month-by-month change of total number of urological surgeries during pandemic and non-pandemic periods. F. Month-by-month change of the number of emergency urological surgeries during pandemic and non-pandemic periods. G. Monthby-month change of the percentage of emergency urological Surgery surgeries during pandemic and non-pandemic periods. H. Month-by-month change of the percentage of emergency use of the urology operating room during pandemic and non-pandemic periods

When the parameters mentioned were evaluated month by month, while the number of applications to emergency department outpatient decreased by $66.38 \%$ in April during the pandemic period compared to the non-pandemic period of 2019 , in the following months, this ratio decreased by $57.05 \%$ in May, $45.61 \%$ in June $31.66 \%$ in July, $18.97 \%$ in August, $13.53 \%$ in September, and $26.28 \%$ in October, it increased by $9.85 \%$ in November (Fig. 1A). No such situation was encountered in the application to urology outpatient (Fig. 1B). The increase in the number of urological consultations, which occurred in the first periods after the declaration of the pandemic, started to decrease after June and decreased to levels like the previous year (Fig. 1C). The percentage of urology consultation reached similar rates to the previous year in the later stages of the pandemic, like the number of applications to emergency department outpatient and urological consultations (Fig. 1D). The total number of urological surgeries was lower during the pandemic compared to the non-pandemic period (Fig. 1E). The number of emergency urological surgeries followed a variable course (Fig. 1F). While the percentage of emergency urological surgeries was higher in the early pandemic period compared to the non-pandemic period of 2019 (April; $0.049 \%-0.014 \%$, May; $0.033 \%-0.009 \%$, June; $0.033 \%-0.013 \%$, July; $0.02 \%$ - $0.009 \%)$, it decreased in August and September (0.015\% -
$0.019 \%, 0.002 \%-0.007 \%$, respectively) and fell below the rates in the 2019 non-pandemic period, and then, it increased again in October and November $(0.029 \%-0.013 \%, 0.017 \%$ $0.002 \%$, respectively) and followed a fluctuating course (Fig. $1 \mathrm{G})$. The percentage of emergency use of the urology operating room also followed a similar graphic course to the percentage of emergency urological surgery (Fig. 1H).

\section{Discussion}

The COVID-19 pandemic has profoundly affected the delivery of health care services around the world. While special and reserved areas were opened for COVID-19 patients, it was aimed to continue routine health care service delivery, especially for emergency and oncological patients. In this process, the European Association of Urology (EAU) defined guiding procedures for urological surgical practices during the pandemic period (7). In accordance with these recommendations, urological surgeries were reorganized, elective-postponable surgeries were postponed, and emergency and oncological surgeries continued, which ensured the continuation of emergency and non-deferrable surgeries along with the decrease in elective surgeries (7-10). Although these recommendations reduced the hospital workload in the short term during the pandemic period, the long-term effects have led to concerns about the complication of postponed surgeries or the need for urgent surgical 
intervention (10-13). During the pandemic period, hospital's outpatient service delivery decreased since national and local administrations made assignments to reduce the hospital workload and to fight against the pandemic.

Our study showed that there was a decrease of $31.05 \%$ in admissions to the emergency department during the 8-month pandemic period compared to the previous year in a similar period. In the studies covering the early stages of the pandemic, it was reported that there was a decrease in admissions to the emergency department $(14,15)$. In a study covering the first 4-week early pandemic period in America, this decrease was found to be $42 \%$, and another study found a decrease of $31 \%-45 \%$ although there was a difference by regions in America $(16,17)$. In our study, while the decrease in admissions to the emergency department was consistent with the literature, when the 8-month period was analyzed month by month, it was observed that the admissions to the emergency department reached similar numbers to the previous periods in the $8^{\text {th }}$ month of the pandemic (medium term), which may be caused by the decrease in admissions to hospitals due to the fear of transmission and anxiety in the society at the beginning of the pandemic, as well as the abuse of emergency departments by those who could not benefit from outpatient health services sufficiently in the early period.

During the pandemic period, a decrease of $67 \%$ was observed in the delivery of urology outpatient services. In their multi-center study, Bozkurt et al. reported this rate as $73 \%$, which may be due to the restrictions imposed by the country's government to reduce social movements, as well as the decrease in hospital admissions due to the fear of Covid transmission in the hospital (6). It can be expected that the decrease in outpatient service delivery will direct people to use emergency departments. Emergency urological surgeries can be expected to increase with the number of urology consultations requested from the emergency department and the percentage of consultations. In our study, an increase of $8.37 \%$ was found in the number of consultations requested. The number of consultations, which was smaller during the first months compared to the non-pandemic period, decreased to numbers like the non-pandemic period after reaching the highest number in the $4^{\text {th }}$ month of the pandemic. In the studies in this regard in the literature, in their multi-center study covering a period of three months, Grasso et al. reported a decrease of $39 \%$ in the number of urology consultations (1). Similarly, in their study covering the 36-day pandemic period, Motterle et al. reported that urology consultations decreased three times during the pandemic period (18). As it is stated in the results of our study and in the literature, there was a decrease in the number of consultations at the beginning of the pandemic. Regarding the contribution of our study to the literature, it showed that the number of consultations would increase during the months when the pandemic continued and would return to non- pandemic periods. Since the number of urology consultations will be affected by the number of patients admitted to the emergency department, the percentage of urology consultation was also examined in our study. It was observed that the percentage of urology consultation was high in the first 4 months of the pandemic, and then, it decreased to the rates like the non-pandemic period after the $6^{\text {th }}$ month.

Regarding urological surgery practices, total urological surgeries decreased by $42.14 \%$ during the pandemic period, like the literature. In the literature, this rate is seen between $18 \%-79.5 \%(5,6,18)$. Although elective surgeries were postponed, these surgeries may be complicated and require immediate intervention during the postponed period. Therefore, an increase in the number of emergency urological surgeries can be expected. Indeed, in our study, this increase was observed in the $7^{\text {th }}$ and $8^{\text {th }}$ months of the pandemic. In our study, although the number of emergency urological surgeries was not statistically significant, an increase of $33.3 \%$ was observed. In the percentage of emergency urological surgeries, an increase from $0.087 \%$ in the non-pandemic period to $0.185 \%$ in the pandemic period and up to $112 \%$ was determined. It was observed that the percentage of emergency use of the urology operating room increased from $2.19 \%$ to $5.06 \%$. In their study, Grasso et al. reported that this ratio increased from $7 \%$ to $11.8 \%$ during the pandemic period (1).

Our study is the first study examining the mid-term experience and reporting the change in urological emergencies, however, it has some limitations, it is a singlecentered study, and our hospital admitted all referrals as a central hospital in the city where it is located, which may also lead to high number and rates of emergency urological surgeries and consultations.

In conclusion, Studies have demonstrated that the pandemic period has a unique variable structure. While the number of outpatient clinics has decreased due to both restrictions and fear of social transmission, emergency department operations and emergency surgical interventions are going on. In this case, different approaches are required in the early stages of the pandemic and in the medium and even long periods. Since emergency departments can be abused for rapid examination in the medium and long periods, it is needed to make different scheduling for emergency department operations and emergency surgeries.

\section{Conflict of interest}

There is no conflict of interest.

\section{Funding}

None.

\section{Acknowledgement}

None to declare.

\section{References}

1. Grasso AAC, Massa G, Castelnuovo M. The Impact of COVID-19 Pandemic on Urological Emergencies: A 
Multicenter Experience on over 3,000 Patients. Urol Int 2021; 105:17-20. https://doi.org/10.1159/000511757.

2. Lu H, Stratton CW, Tang YW. Outbreak of pneumonia of unknown etiology in Wuhan, China: The mystery and the miracle. J Med Virol 2020; 92:401-2. doi: 10.1002/jmv.25678.

3. Huang C, Wang Y, Li X, Ren L, Zhao J, Hu Y, et al. Clinical features of patients infected with 2019 novel coronavirus in Wuhan, China. Lancet. 2020; 395(10223):497-506. doi: 10.1016/S0140-6736(20)30183-5.

4. World Health Organization. WHO director-General's opening remarks at the media briefing on COVID-19 - 11 March 2020. World Health Organization. [Internet]. 2020 [updated 2020 March 11; cited January 10, 2021]. Available from: https://www.who.int/dg/speeches/detail/who-director-generals-opening-remarks-at-the-media-briefing-on-covid-19---11march-2020.

5. Teoh JY, Ong WLK, Gonzalez-Padilla D, Castellani D, Dubin JM, Esperto F, et al; UroSoMe Working Group. A Global Survey on the Impact of COVID-19 on Urological Services. Eur Urol. 2020 Aug;78(2):265-275. doi: 10.1016/j.eururo.2020.05.025. Epub 2020 May 26.

6. Bozkurt O, Sen V, Irer B, Sagnak L, Onal B, Tanidir Y, et al; Study Group of the Society of Urological Surgery. Nation-wide analysis of the impact of Covid-19 pandemic on daily urology practice in Turkey. Int J Clin Pract. 2021 Apr;75(4):e13735. doi: 10.1111/ijcp.13735. Epub 2020 Nov 3.

7. Ribal MJ, Cornford P, Briganti A, Knoll T, Gravas S, Babjuk $\mathrm{M}$, et al. European Association of Urology Guidelines Office Rapid Reaction Group: An Organisation-wide Collaborative Effort to Adapt the European Association of Urology Guidelines Recommendations to the Coronavirus Disease 2019 Era. Eur Urol. 2020;78(1):21-8. doi.org/10.1016/j.eururo.2020.04.056.

8. Stensland KD, Morgan TM, Moinzadeh A, Lee CT, Briganti A, Catto JWF, et al. Considerations in the Triage of Urologic Surgeries During the COVID-19 Pandemic. Eur Urol. 2020 Jun;77(6):663-666. doi: 10.1016/j.eururo.2020.03.027. Epub 2020 Apr 9.

9. Simonato A, Giannarini G, Abrate A, Bartoletti R, Crestani A, De Nunzio $C$, et al. Clinical pathways for urology patients during the COVID-19 pandemic. Minerva Urol Nefrol. 2020;
72(3):376-83. doi.org/10.23736/S0393-2249.20.03861-8.

10. Ficarra V, Novara G, Abrate A, Bartoletti R, Crestani A, De Nunzio C, et al F; Research Urology Network (RUN). Urology practice during the COVID-19 pandemic. Minerva Urol Nefrol. 2020 Jun;72(3):369-375. doi: 10.23736/S0393-2249.20.038461. Epub 2020 Mar 23

11. Uribarri J. Chronic kidney disease and kidney stones. Curr Opin Nephrol Hypertens 2020; 29:237-42. doi.org/10.1097/MNH.0000000000000582.

12. Proietti S, Gaboardi F, Giusti G. Endourological stone management in the era of the COVID-19. Eur Urol. 2020;78(2):131-3. doi.org/10.1016/j.eururo.2020.03.042.

13. Amparore D, Campi R, Checcucci E, Sessa F, Pecoraro A, Minervini A, et al. Forecasting the Future of Urology Practice: A Comprehensive Review of the Recommendations by International and European Associations on Priority Procedures During the COVID-19 Pandemic. Eur Urol Focus. 2020 Sep 15;6(5):1032-1048. doi: 10.1016/j.euf.2020.05.007. Epub 2020 May 31 .

14. Cicerello E, Mangano MS, Cova G, Zordani A. Urological emergency activities during COVID-19 pandemic: Our experience. Arch Ital Urol Androl. 2020; 92(4). doi.org/10.4081/aiua.2020.4.282.

15. Giamello JD, Abram S, Bernardi S, Lauria G. The emergency department in the COVID-19 era. Who are we missing? Eur J Emerg Med. 2020;27(4):305-6. doi.org/10.1097/MEJ.0000000000000718.

16. Hartnett KP, Kite-Powell A, DeVies J, Coletta MA, Boehmer TK, et al; National Syndromic Surveillance Program Community of Practice. Impact of the COVID-19 Pandemic on Emergency Department Visits - United States, January 1, 2019May 30, 2020. MMWR Morb Mortal Wkly Rep. 2020 Jun 12;69(23):699-704. doi: 10.15585/mmwr.mm6923el.

17. Boserup B, McKenney M, Elkbuli A. The impact of the COVID-19 pandemic on emergency department visits and patient safety in the United States. Am J Emerg Med. 2020; 38(9):1732-6. doi.org/10.1016/j.ajem.2020.06.007.

18. Motterle $G$, Morlacco A, Iafrate $M$, Bianco M, Federa G, Xhafka $O$, et al. The impact of COVID-19 pandemic on urological emergencies: a single-center experience. World J Urol. 2020 May 23:1-5. doi: 10.1007/s00345-020-03264-2. Epub ahead of print. 